\title{
The Effect of Spatial Clustering of Motor Fibers in a Nerve Fascicle on Force Recruitment during Electrical Stimulation
}

\author{
Jos P.A. Smit, Wim L.C. Rutten, Member, IEEE, Theo A. Frieswijk, \\ Tom H. Rozijn, and Ronald Busschers \\ Department of Biomedical Engineering, Faculty of Electrical Engineering, \\ University of Twente, P.O. Box 7500 AE Enschede, The Netherlands
}

\begin{abstract}
Force recruitment curves have been determined using stimulation with an intrameural wire electrode. The recruitment curves vary with the position of the electrode in the fiber bundle. The results seem to support previous findings which indicated that the low density of nodes of Ranvier and a possible clustering of motor fibers within the fiber bundle might have a major impact on the efficacy of intraneural electrical stimulation.
\end{abstract}

\section{INTRODUCTION}

In electrical nerve stimulation selectivity can be increased using intraneural electrodes. In a theoretical analysis Rutten et al. derived a cubic dependence of muscle force on stimulus current amplitude: $F \sim \mathrm{I}^{3}[1]$. They used a local approach and assumed a uniform distribution of nodes of Ranvier and an isotropic medium.

Animal experiments confirmed this cubic dependence; however, steeper dependencies were also found. In experiments carried out by Meier the steepness of the experimental curves was generally higher than 3 [2]. (The steepness of a recruitment curve is defined as the power of $I$ that $F$ is proportional to, e.g. when $F \sim I^{p}$ then the steepness is p.) Meier suggested that this might be caused by the fact that the distribution of $\alpha$ motor fibers inside the fascicle is not uniform as was assumed in the theoretical analysis. Therefore, clustering of the $\alpha$ motor fibers in a subbundle of the fascicle might occur.

\section{SIMULATIONS}

The model used to simulate intraneural stimulation is an extension of the model presented by Meier et al. [3]. It incorporates the possibility of clustering of $\alpha$ motor fibers in a subbundle of the fascicle. Fig. 1 shows simulation results for the case of a circular subbundle of $250 \mu \mathrm{m}$ diameter inside a fascicle of $500 \mu \mathrm{m}$ diameter. The fibers are distributed uniformly over the cross-section of the subbundle. Thus, the $\alpha$ motor fibers are clustered in a region of only one fourth of the total fascicle cross-section. Results are shown for two electrode positions: one in the middle of the subbundle [Fig.1(a) and 1(c)] and one outside the subbundle [Fig. 1(b) and 1(d)].

\section{0-7803-0785-2/92\$03.00 @IEEE}

From Fig. 1 it can be seen that the steepness of the recruitment curves depends on the position of the electrode relative to the cluster: the steepness increases for an electrode moving from a position inside the cluster to a position outside the cluster (electrode 1 resp. 2, see inset in Fig. 1). Fig. 1 also shows that the steepness increases with decreasing $\sigma_{\mathrm{e}}$.

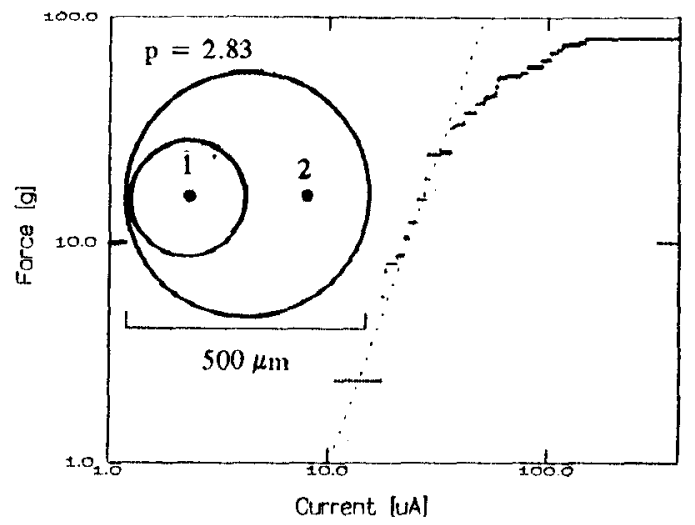

(a)

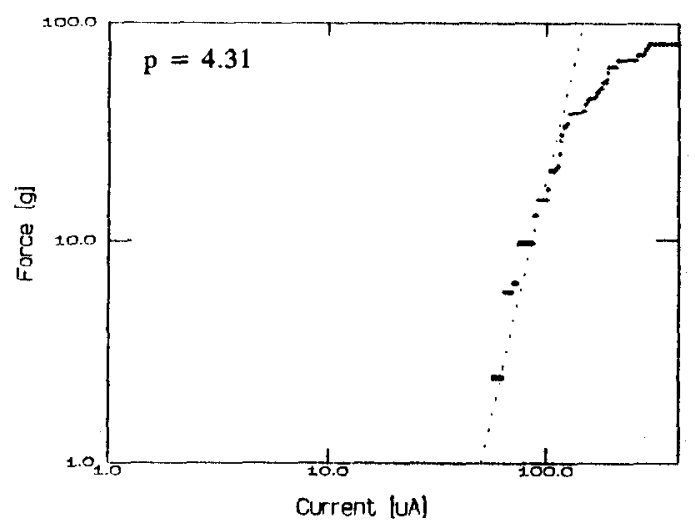

(b)

Fig. 1 (continued) 


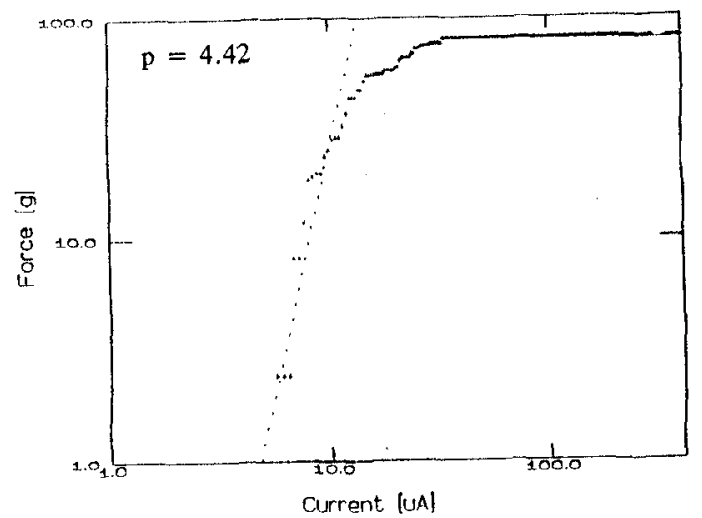

(c)

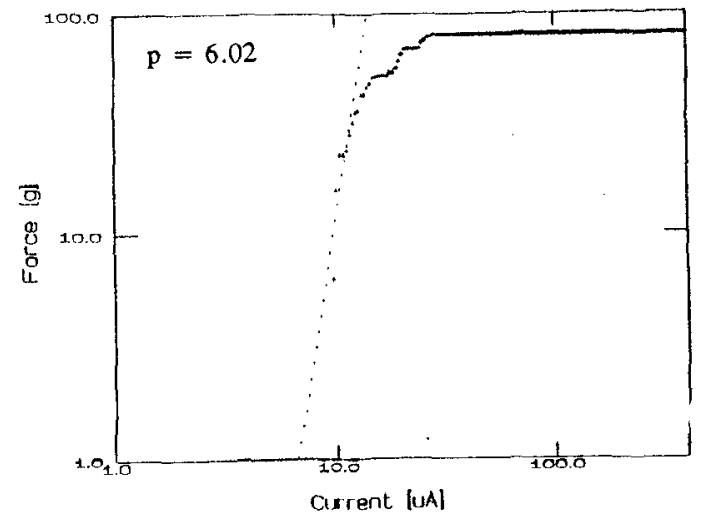

(d)

Fig. 1. Simulated recruitment curves for monopolar stimulatation of EDL muscle in the case of clustering of $\alpha$ motor fibers. The steepness $p$ is shown for every curve. The effect of clustering of $\alpha$ motor neurons on the recruitment curves resulting from stimulation with an electrode inside the cluster [(a) and (c)] and with an electrode outside the cluster [(b) and (d)] are shown. (Electrode positions are shown in the inset of (a).) For both electrodes recruitment curves are shown for an external medium conductivity $\sigma_{\mathrm{e}}=0.1 \Omega^{-1} \mathrm{~m}^{-1}[(\mathrm{a})$ and $(\mathrm{b})]$ and $\sigma_{\mathrm{e}}=0 \Omega^{-1} \mathrm{~m}^{-1}[(c)$ and (d)].

\section{EXPERIMENTS}

During acute experiments a wire electrode (diameter $=25 \mu \mathrm{m}$, active tip area $=2.78 \cdot 10^{-9} \mathrm{~m}^{2}$ ) was inserted in the intact peroneal nerve of the right hindleg of anaesthetised Wistar rats. Monophasic rectangular current pulses of $100 \mu$ s duration were delivered in order to stimulate $\alpha$ motor fibers of the extensor digitorum longus muscle (EDL) and the tibialis anterior muscle (TA). The distal tendons were cut and connected to a force transducer. The elicited twitch contraction forces were measured under isometric conditions.

Typical recruitment curves are shown in Fig. 2. This figure contains pooled data from three experiments for different electrode positions. The curves were obtained from the EDL [Fig. 2(a)], the TA [Fig. 2(b)], and the EDL and TA tied together [Fig. 2(c)].

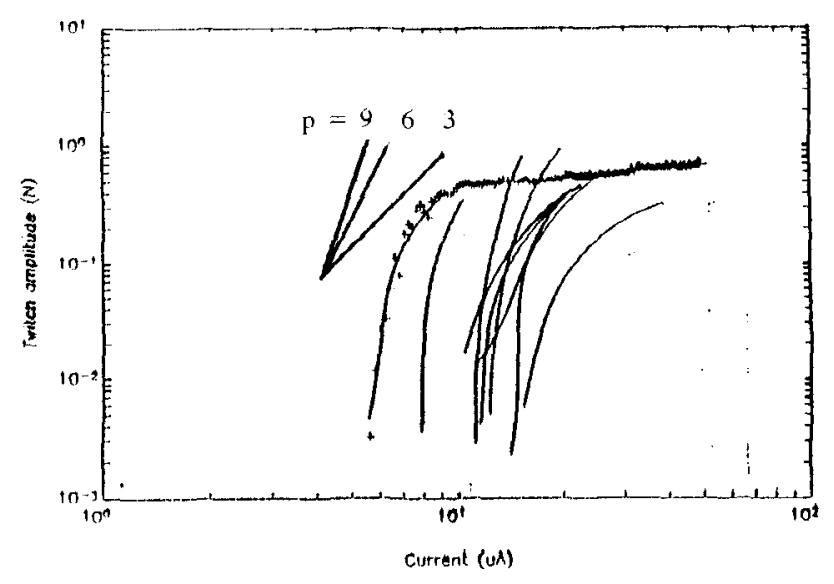

(a)

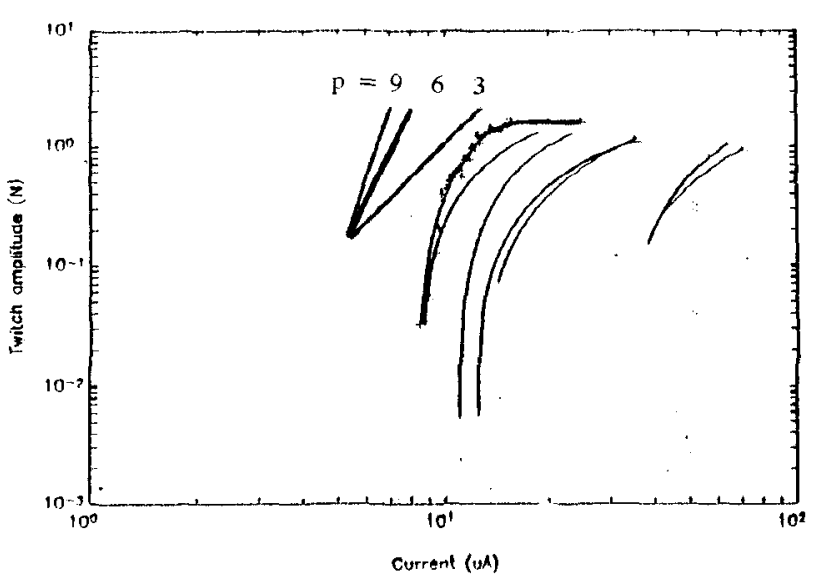

(b)

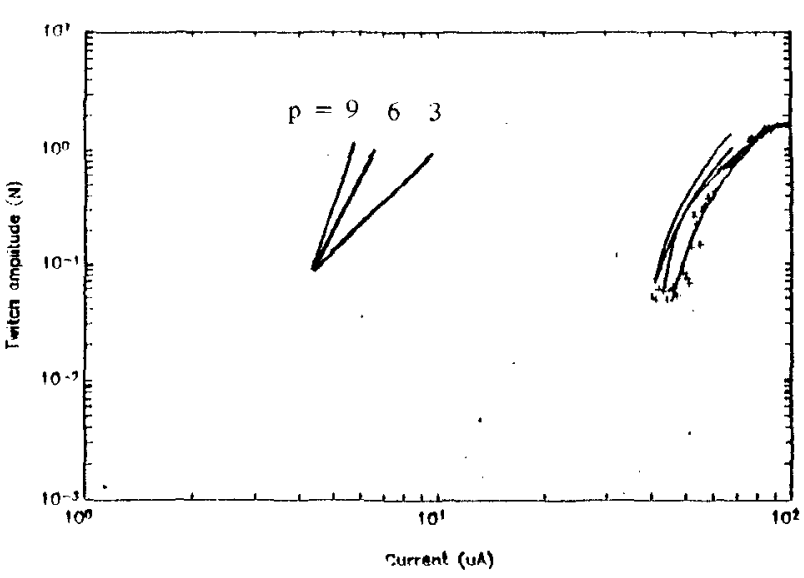

(c)

Fig. 2. Pooled recruitment curves from three experiments obtained from the EDL [(a)], the TA [(b)], and the EDL and TA tied together [(c)]. The curves are smooth fits of the experimental data. In every plot one experimental data series is shown as well as three reference lines corresponding to steepnesses $\mathrm{p}=3,6$, and 9 . 
The results in Fig. 2 show that single motor units can be stimulated, since force levels as low as $2 \cdot 10^{-3} \mathrm{~N}$ can be observed. Generally the onset of recruitment is as low as $10 \mu \mathrm{A}$ to $20 \mu \mathrm{A}$. However, higher thresholds also occur. This threshold variation might be caused by a variation in the distance between the electrode and the nearest $\alpha$ motor fiber.

Fig. 2 also shows that the steepness of the recruitment curves changes when stimulation current increases from the threshold level to higher values. In the low force range steepnesses as high as 9 can be observed, whereas in the middle force range the steepnesses decrease to values as low as 3. This effect was also observed by Meier [2].

\section{Discussion}

The simulation results presented in Fig. 1 show that the steepness of the recruitment curves depends on the position of the stimulation electrode: for an electrode inside the cluster the steepness is higher than for an electrode outside the cluster.

Fig. 2 shows that the steepness of the experimental recruitment curves varies gradually rather than having a constant value. In the low force range steepnesses as high as 9 are found, which is higher than those found from the simulations. This might be caused by the fact that the cluster is restricted to an even smaller region of the fascicle than was assumed in the simulations. Another explanation might be that during the experiments the nerve is more or less isolated from the body fluids. This implies that the external medium conductivity $\sigma_{e}$ in reality is smaller than the $0.1 \Omega^{-1} \cdot \mathrm{m}^{-1}$ assumed in the simulations. Additional simulations with $\sigma_{c}=0 \Omega^{-1} \cdot \mathrm{m}^{-1}$ indeed show higher steepnesses [Fig. 1(c) and 1(d)]. Also, the onset of recruitment decreases to values that compare favorably well to those observed from the experiments.

Additional simulations and experiments have to be done to further assess the influence of the distribution of $\alpha$ motor fibers within the fiber bundle on the efficacy of intraneural electrical stimulation.

\section{REFERENCES}

[1] W.L.C. Rutten, H.J, van Wier, and J.H.M. Put, "Sensitivity and selectivity of intraneural stimulation using a silicon electrode array," IEEE Trans. Biomed. Eng., vol. 38, pp. 192-198, Feb. 1991.

[2] J.H. Meier, Selectivity and design of neuro-electronic interfaces, Ph.D. thesis, University of Twente, Enschede, The Netherlands, 1992.

[3] J.H. Meier, W.L.C. Rutten, A.E. Zoutman, H.B.K. Boom, and P. Bergveld, "Simulation of fiber selective neural stimulation using intrafascicular electrodes," IEEE Trans. Biomed. Eng., vol. 39, pp. 122-134, Feb. 1992. 